\title{
Hybrid microincision vitrectomy surgery combined with 20-gauge silicone cannulas for use with 20-gauge horizontal scissors in diabetic tractional retinal detachment
}

\author{
This article was published in the following Dove Press journal: \\ Clinical Ophthalmology \\ 31 July 2013 \\ Number of times this article has been viewed
}

\section{Keiko Yamada' \\ Takatoshi Maeno ${ }^{2}$ \\ Mitsunori Yamada ${ }^{3}$ \\ 'Department of Ophthalmology, Kyoto Prefectural University of Medicine, Kyoto, Japan; ${ }^{2}$ Department of Ophthalmology, Toho University Sakura Medical Center, Chiba, Japan; ${ }^{3}$ Yamada Eye Clinic, Tokushima, Japan}

Correspondence: Keiko Yamada Yamada Eye Clinic, 2-3I, Nakatorimachi, Tokushima,Tokushima 770-0844 Japan Email yamada@eye-yamada.com
Purpose: To verify the utility and preliminary safety of a 20-gauge silicone cannula for use with 20-gauge horizontal scissors delamination during microincision vitrectomy surgery (MIVS).

Methods: Thirty-eight eyes in 35 consecutive patients with diabetic tractional retinal detachment, who underwent MIVS between April 2010 and March 2012 and were followed for 3-24 months, were retrospectively assessed using a chart review. Twenty-gauge scissors delamination through a silicone cannula, with an additional 20-gauge port as a hybrid, was primarily selected when treating thick and rigid fibrovascular membranes, including fluctuating vessels over the detached retina near the macula. The main outcome measures included the proportion of patients treated with this hybrid method, the postoperative visual acuity, and the incidence of complications.

Results: Compared with the 26 eyes treated with MIVS only, 12 eyes (32\%) required a hybrid technique with the use of 20 -gauge instruments through a silicone cannula in addition to MIVS. Two patients underwent additional surgery. Temporary silicone oil tamponade was performed in one case of retinotomy and one case of schizophrenia. The mean visual acuity (logarithm of the minimum angle of resolution [ $\log \mathrm{MAR}]$ ) improved from $1.43 \pm 0.85$ to $0.72 \pm 0.47$ at the last follow-up visit. No patients exhibited worsening of their visual acuity postoperatively. No sclerotomy-related complications were recorded during the intraoperative or postoperative periods.

Conclusion: Hybrid MIVS combined with a 20-gauge silicone cannula for use with 20-gauge horizontal scissors in diabetic tractional retinal detachment eyes is useful and safe due to the reduced risk of sclerotomy-related retinal breaks. This procedure is a reasonable option when performing complex surgery for diabetic vitrectomy.

Keywords: small gauge vitrectomy, cannulated vitrectomy system, cutter delamination, scissor delamination, 20-gauge instruments, sclerotomy retinal tears

\section{Introduction}

The recent establishment of microincision vitrectomy surgery (MIVS) with 27-, 25-, or 23-gauge instrumentation has provided advantages over traditional 20-gauge surgery. ${ }^{1}$ Small vitreous cutters are increasingly used for the removal and segmentation of fibrovascular membranes in patients with diabetic tractional retinal detachment (DTRD). However, cutter instrumentation cannot be used in place of scissors delamination in all cases. Some surgeons select horizontal scissors in refractory cases, such as those involving broad-based table-top type DTRD. ${ }^{2}$ That is, it is sometimes necessary to mix small- and large-gauge vitrectomy instrumentation, as 
some instruments, including sharply curved scissors, are only available in 20 -gauge sizes. ${ }^{3}$

Iatrogenic retinal tears associated with pars plana entry sites are one of the most serious complications of vitrectomy surgery. A statistically significant reduction in the rate of intraoperative retinal breaks has been reported in patients undergoing cannulated pars plana vitrectomy (PPV), including that performed with 19-, 20-, 23-, and 25-gauge systems. ${ }^{4,5}$ The use of a 20-gauge silicone cannula system has also been introduced in standard, three-port, 20-gauge PPV. ${ }^{6}$ The cannula, which is fabricated from silicone, is self-sealing and allows for the passage of sharply angulated intraocular instruments that cannot pass through other solid metal cannulas. Surgeons can utilize this flexible 20-gauge cannula to perform hybrid MIVS, ie, vitrectomy completed with 25-gauge cutters, combined with 20 -gauge instruments, leading to a lower risk of sclerotomy-related tears. The aim of this study was to elucidate the utility and safety of performing hybrid MIVS using a 20-gauge silicone cannula in 20-gauge horizontal scissors delamination during DTRD surgery.

\section{Methods}

Of the 38 eyes in 35 consecutive patients with DTRD, 12 eyes in 11 patients who underwent hybrid MIVS were enrolled in this study. The patients included four females and seven males (mean age: $58.5 \pm 6.6$ years) who underwent MIVS combined with a 20-gauge silicone cannula between April 1, 2010 and March 31, 2012. All patients were from the same hospital and had type II diabetes with systemic complications, such as hemodialysis. The institutional review board of the Yamada Eye Clinic approved the current study, and informed consent was obtained from all of the participants in accordance with the Declaration of Helsinki. Proliferative diabetic retinopathy (PDR) was classified based on the Early Treatment Diabetic Retinopathy Study. ${ }^{7}$ The surgeries were performed by two experienced vitreous surgeons (TM and MY). Each eye was judged to have DTRD in PDR based on the medical records of the hospital. Indirect slit-lamp ophthalmoscopy (75 SL; Zeiss) with high power convex lenses (Volk Optical, Inc, Mentor, OH, USA) was used to diagnose DTRD, along with an ultrasonic B-mode scanner (UD-1000; TOMEY GmbH, Erlangen, Germany) and Cirrus HD-OCT (Zeiss Meditec, Dublin, CA, USA).

All patients received local retrobulbar anesthesia during surgery. Phacoemulsification, aspiration, and implantation of an acrylic foldable intraocular lens were performed simultaneously through a temporal sclero-corneal incision in patients with preexisting cataracts. Conjunctival peritomy with MIVS was used as a prelude in cases of DTRD in this study in order to facilitate adequate scleral indentation and prevent insufficient vitreous shaving. The setting for MIVS was based on a 23-gauge two-step entry through an angled incision introduced by Echardt, ie, the oblique perpendicular method. ${ }^{8}$ At a distance of $3.5 \mathrm{~mm}$ from the limbus, a scleral tunnel was made with a 23 -gauge, $45^{\circ}$-angled microvitreoretinal (MVR) blade (Alcon Laboratories, Inc, Fort Worth, TX, USA) inserted at an angle of approximately $30^{\circ}$ onto the scleral surface. A blunt trocar inserter was passed through the cannula and inserted into the incision. The blunt trocar inserter was removed, and the infusion cannula was connected to the inferior temporal tunnel. Two other cannulas were then placed at the superior quadrant after making incisions using the same method. Vitrectomy was performed using the standard 23-gauge instruments, including a backflush needle and a diamond-dusted sweeper (DORC), except for the 25-gauge cutter, with the ACCRUS 800 surgical system (Alcon Laboratories, Inc) followed by 25 -gauge cutter delamination or segmentation through a 23-gauge solid metal cannula. The internal limited membrane (ILM) on the macula was, if necessary, peeled away using 23-gauge microforceps (AU-2286.K-06; DORC). When thick and rigid fibrovascular membranes, including fluctuating vessels over the detached retina, were located near the macula, 20-gauge scissors delamination through a silicone cannula in another port was intraoperatively selected. That is, a scleral plug was inserted into one of the 23-gauge cannulas. A perpendicular incision was made through the sclera $3.5 \mathrm{~mm}$ away from the limbus at approximately the 10 or 2 o'clock position using a 20-gauge MVR blade. ${ }^{9}$ Next, a 20 -gauge silicone cannula with a closed tip was inserted into the eye with the assistance of an inserter (ME Technica Co, Ltd, Tokyo, Japan), and the cannula tip was cut in a cross using the same 20-gauge MVR blade. Finally, the procedure used $55^{\circ}$ angulated Sutherland rotatable 20-gauge horizontal intraocular microscissors modified by Charles (612.22; Grieshaber, Switzerland) (Figure 1A and B). All surgeries used prism contact lenses and a wide angle viewing system (HRX; Volk Optical Inc). The intravitreous injection of triamcinolone acetonide was used to visualize the vitreous gel and identify the location of vitreoretinal adhesion. Fluid-gas exchange was implemented following endolaser photocoagulation with a premixed nonexpansible concentration of $20 \%$ sulfur hexafluoride or silicone oil (1,000 cs). Endoillumination with gentle scleral indentation helped to identify the peripheral retinal 


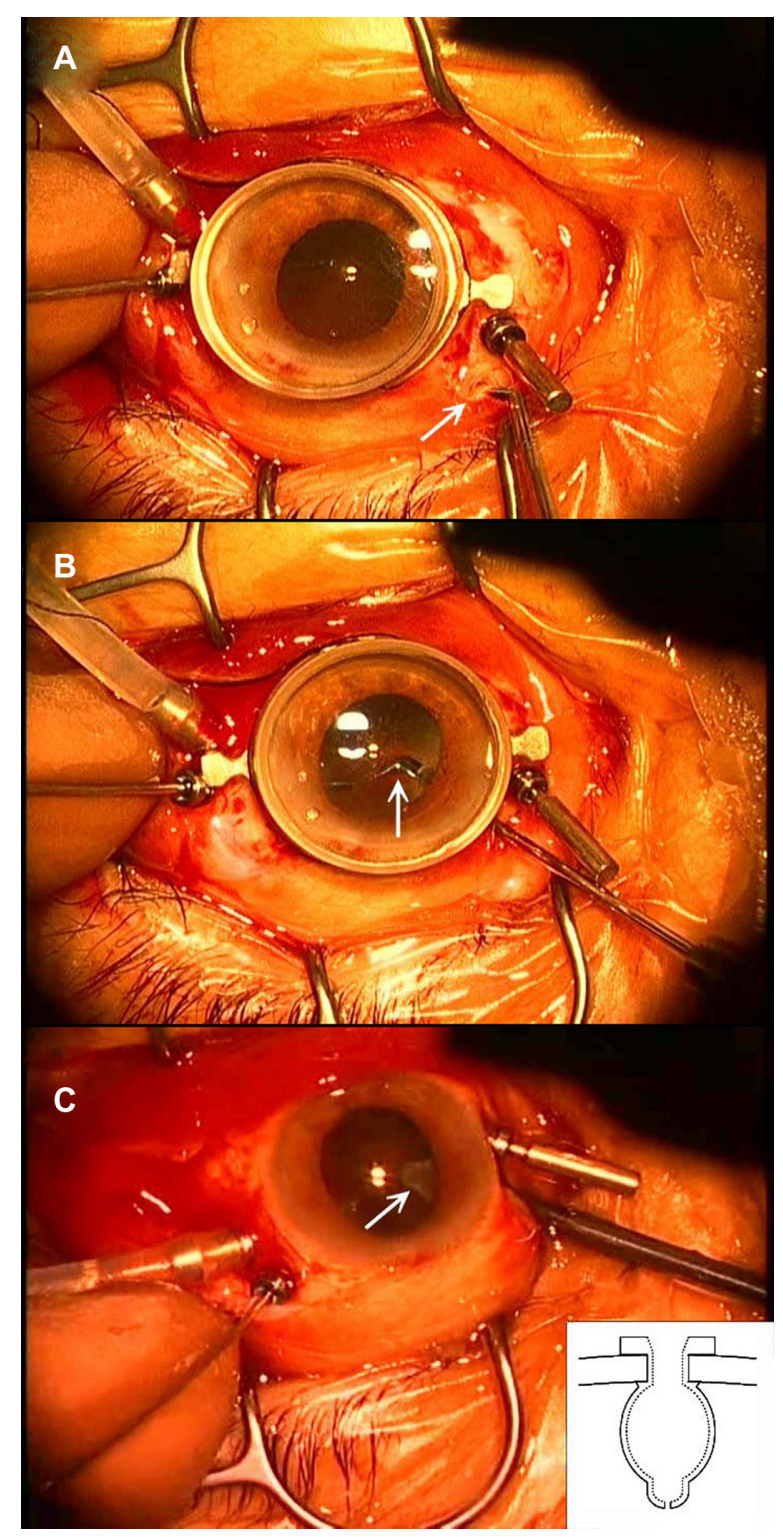

Figure I Intraoperative view of the hybrid microincision vitrectomy surgery system using a 20-gauge silicone cannula for horizontal scissors delamination. (A) A 20-gauge silicone cannula port (arrow) placed at approximately the 10 o'clock position with 20-gauge horizontal scissors placed nearby. (B) 20-gauge horizontal scissors (arrow) inserted into the vitreous cavity for delamination. (C) A spindle-shaped 20-gauge silicone cannula whose tip is closed (arrow) at the time of scleral indentation. The silicone cannula is illustrated in the inset.

pathologic features at the end of the surgery (Figure 1C). All inserted instruments were removed, and the surgery was completed usually without sutures, except to close the 20-gauge silicone cannula port, which was sutured with 8-0 VICRYL (TG 140-8; Ethicon Inc, Somerville, NJ, USA). Topical antibiotic ointment was applied to the cul-de-sac, and the eye was patched and shielded. The patients were instructed to maintain a prescribed posture for several hours over 3-7 days.

\section{Results}

The clinical data of the 12 eyes in which 20-gauge scissors delamination was combined with MIVS are shown in Table 1. Cataract surgery was performed in seven eyes (cases 1, 2, 3, 4, 5, 10, and 12) along with intraocular lens implantation. The DTRD range and macula status of each PDR case was directly confirmed during surgery and recorded in the chart postoperatively by the surgeon. One male patient with diabetic gangrene (case 1) required relaxing retinotomy followed by scleral buckling and silicone oil tamponade. One female schizophrenic patient (case 12) developed retinal redetachment, and silicone oil injection was necessary during an additional surgery. The silicone oil was removed after 3 months. Mean follow-up duration was $1.9 \pm 7.4$ months (range: 3-24 months).

A 25-gauge vitreous cutter was used during MIVS to cut and remove the dense vitreous hemorrhage, grasp, peel, and dissect the fibrovascular membranes, and gently compress the bleeding point for hemostasis. After removing the hazy optic media, as shown in Figure 2B, 20-gauge horizontal scissors were used as needed to address lesions with thick and rigid fibrovascular membranes, particularly in the macular area. The exact indications for the use of 20-gauge scissors delamination depended on the individual preference of each surgeon. The ILM on the macula was removed in cases 3 (OD) and 6 to prevent recurrent proliferation of the epiretinal membranes (ERMs). Twenty-three-gauge sclerotomy was principally self-sealed after surgery in all cases, unless leakage was noted (case 1), without suture placement, except for the 20-gauge silicone cannula port. Anatomic success was achieved in all cases, including the additional surgeries in cases 2 and 12, with the successful removal of the ERMs, ILM, or both or reattachment of the detached retina. The visual acuity (VA) of each of the 12 eyes at baseline and at the last follow-up is shown in Table 1 . The preoperative mean VA improved from $1.43 \pm 0.85$ to $0.72 \pm 0.47 \log$ MAR (logarithm of the minimum angle of resolution) at the last follow-up visit. The postoperative best-corrected VA was restored to at least the preoperative level during the follow-up period in all cases. The patient in case 3 (OS) achieved a good VA of $0.045 \log$ MAR (20/22) following the removal of thick, clouded media due to preoperative dense vitreous hemorrhage (Figure 2A and C). Cases 6 and 9 resulted in limited visual improvement due to longstanding or severe macular disease. The systemic and operative complications are also shown as notes in Table 1. No sclerotomy-related complications were documented 
Table I Characteristics of the I2 DTRD eyes treated with hybrid MIVS using 20-gauge horizontal scissors through a silicone cannula

\begin{tabular}{|c|c|c|c|c|c|c|c|c|}
\hline \multirow[t]{2}{*}{ Case no } & \multirow[t]{2}{*}{ Age/gender } & \multirow[t]{2}{*}{ Lens status } & \multirow{2}{*}{$\begin{array}{l}\text { Quadrant of } \\
\text { DTRD, range }\end{array}$} & \multirow{2}{*}{$\begin{array}{l}\text { Macula } \\
\text { status }\end{array}$} & \multicolumn{2}{|c|}{ VA (logMAR) } & \multirow{2}{*}{$\begin{array}{l}\text { Follow-up } \\
\text { months }\end{array}$} & \multirow[t]{2}{*}{ Notes } \\
\hline & & & & & Preop & Postop & & \\
\hline I & $69 / M$ & Phakia & Superior arcade & Off & 2.0 & 1.0 & 23.5 & Retinotomy, SO \\
\hline 2 & $59 / M$ & Phakia & Superior arcade & On & 0.7 & 0.39 & 10 & Re-RD \\
\hline 3 & $54 / F$ & Phakia & Temporal arcade & Off & 3.0 & 0.045 & 24 & OS \\
\hline 4 & $54 / F$ & Phakia & Disc nasal & On & 1.0 & 0.7 & 14 & OD \\
\hline 5 & $68 / M$ & Phakia & Infero-temporal & On & 0.52 & 0.39 & 16 & \\
\hline 6 & $60 / M$ & IOL & Temporal & On & 0.7 & 0.7 & 12 & Hemodialysis \\
\hline 7 & $61 / M$ & IOL & Superior & On & 1.0 & 0.7 & 18 & Prior vitrectomy \\
\hline 8 & $61 / M$ & IOL & Infero-temporal & On & 0.39 & 0.15 & 10 & Cerebral infarction \\
\hline 9 & $65 / F$ & IOL & Superior & Off & 1.7 & 1.7 & 4 & Cerebral infarction \\
\hline 10 & $63 / M$ & Phakia & Infero-temporal & Off & 1.7 & 0.52 & 3.5 & \\
\hline II & $76 / M$ & IOL & Total & Off & 3.0 & 1.4 & 3 & Hemodialysis \\
\hline 12 & $55 / F$ & Phakia & Supero-nasal & Off & 1.4 & 1.0 & 5 & Schizo, Re-RD, SO \\
\hline
\end{tabular}

Notes: VA is represented as the logMAR and the pre- and postoperative changes in the best-corrected VA; logMAR 3.0; hand movement at 2ft.

Abbreviations: DTRD, diabetic tractional retinal detachment; IOL, intraocular lens; logMAR, logarithm of the minimum angle of resolution; MIVS, microincision vitreous surgery; Re-RD, recurrent retinal detachment; Schizo, schizophrenia; SO, silicone oil; VA, visual acuity; OD, right eye; OS, left eye.

during the intraoperative or postoperative periods in the chart review of this study.

\section{Discussion}

In the vast majority of DTRD cases, ERMs are highly adherent with ischemia caused by poor perfusion. Attempts to peel or strip such membranes using picks or similar tools are prone to inducing iatrogenic retinal breaks. ${ }^{2}$ Frequent membrane delamination requires repeated entry into the eye with multiple instruments as the fibrovascular traction is carefully excised from the surface of the retina. The repeated exchange of instruments, such as horizontal scissors, easily

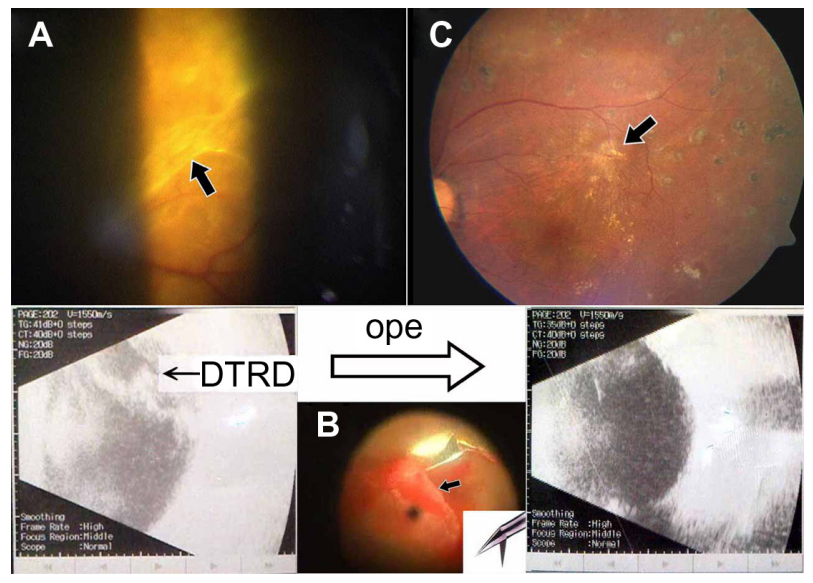

Figure 2 Preoperative and postoperative fundus views in a patient with DTRD treated with hybrid microincision vitrectomy surgery (case 3). (A) A preoperative fundus photograph of the ERMs (arrow) and an ultrasound echogram (bottom left) of DTRD. (B) ERM (arrow) delamination using $55^{\circ}$ angulated scissors (shown in the inset) in a still frame of surgical videography, presented as an inverted fundus image. (C) A postoperative fundus photograph showing treated ERMs (arrows) and an ultrasound echogram (bottom right) of the reattached retina.

Abbreviations: DTRD, diabetic tractional retinal detachment; ERM, epiretinal membrane. causes vitreous traction on entry sites, resulting in retinal ora tears. The incidence of sclerotomy-related retinal breaks is higher in cases of complex DTRD; however, fortunately, no instance of this kind was observed in this study. The cannula system protects the vitreous base by allowing for easier entry of instruments into the eye, thus resulting in a lower incidence of herniation of the vitreous outside of the port site and possibly better removal of the vitreous at the location of the sclerotomy. Territo et al demonstrated a statistically significant decrease in the incidence of sclerotomy tears $(1.0 \%$ versus $7.7 \%, P<0.05$ ) in the 19 -gauge cannulated system group (41 eyes) relative to that observed in the noncannulated group ( 36 eyes) in their series of 77 eyes undergoing pars plana vitrectomy. ${ }^{4}$ Covert et al reported that their study of 426 eyes was the first to find a statistically significant reduction $(3.3 \%$ versus $23.0 \%, P<0.01)$ in the rate of intraoperative retinal breaks using transconjunctival cannulated PPV, including the 20-, 23-, and 25-gauge systems. ${ }^{5}$

Small-gauge systems, such as MIVS, have become popular, even in cases of proliferative vitreous retinopathy (PVR). MIVS using 25-gauge cutters can be used to repair retinal detachment complicated by PVR as well as or better than traditional 20 -gauge vitrectomy surgery. ${ }^{10}$ In this study, 25 -gauge cutters were used through a 23 -gauge cannula system since 25 -gauge cutters are useful in terms of delicate manipulation during membrane peeling and reduced inflammation for preventing progression to PVR, while 23-gauge cutters are often necessary for dealing with rigid tissues or lens particles. Reduced postoperative inflammation enables the patient to achieve a good VA by means of the early PPV, such as in case 3 (OS). Although vitreous cutters are increasingly being used for removal and segmentation of 
ERMs in cases of DTRD, cutter delamination cannot replace scissors delamination in all cases. ${ }^{2}$ The limitations of MIVS can be overcome by enlarging one port to the 20-gauge size for some tasks. In the current series, the procedures used to treat DTRD in all 38 eyes began with MIVS using 25-gauge cutter delamination, and 12 cases further required the use of a hybrid technique, ie, vitrectomy completed not only with 25 -gauge instruments, but also with 20-gauge instruments by creating another 20 -gauge port. The 20 -gauge silicone cannula is self-sealing to prevent intraoperative hypotony, eliminating worry regarding accidental dislocation of a scleral plug during the procedure and allowing for the passage of strongly curved intraocular instruments that cannot pass through other solid metal cannulas. ${ }^{6}$ In this study, 20-gauge $55^{\circ}$-angled horizon scissors were used because they fit easily through the silicone cannula due to the gauge and curvature. There are three reasons for using 20-gauge horizontal scissors. First, using 23- to 25-gauge curved scissors is risky because the scissors can impale the retina with their tips due to the short length of the blade, particularly when used by inexperienced surgeons. Second, it is difficult to control aspiration during cutter delamination in order to prevent accidental retinal breaks. Third, the bimanual technique is often used to incorrectly lift ERMs too high, thus leading to the occurrence of iatrogenic retinal tears. Performing careful scissors delamination is safer, even if the one-hand method is associated with difficulties in managing fluctuating membranes, especially around the macular area. Moreover, the use of a silicone cannula enables 20-gauge horizontal scissors delamination to be performed with a lower risk of sclerotomy-related breaks.

Caution is thus warranted when evaluating the merits of the hybrid system introduced in this study, since the findings are based on limited cases. Further studies with additional various cases are needed for statistical analyses. Nevertheless, the present findings suggest that the flexibility and fragility of the shaft rigidity of small-gauge instruments continue to be associated with problems, including higher costs. The increased flexibility of smaller gauge instruments limits the ability to use long, oblique incisions due to deformation of the shaft when the oblique entry is brought into the perpendicular position that is most convenient when performing vitrectomy. ${ }^{3}$ Meanwhile, the system presented in this study allows for the use of many traditional 20-gauge vitrectomy instruments to overcome these problems. ${ }^{3,11}$ Flexible silicone cannulas are therefore recommended for use in the atraumatic management of 20-gauge scleral incisions.

\section{Acknowledgment}

Authors wish to thank A Nishimura, MD, PhD for kindly providing the silicone cannulas.

\section{Disclosure}

The authors report no conflicts of interest in this work.

\section{References}

1. Oshima Y, Wakabayashi T, Sato T, et al. A 27-gauge instrument system for transconjunctival sutureless microincision vitrectomy surgery. Ophthalmology. 2010;117:93-102.

2. Charles ST, Calzada J, Wood B. Combined 25-gauge and 20-gauge vitrectomy. pp 107-108. Diabetic traction retinal detachment. pp 179-183. In: Vitreous Microsurgery. 5th ed. Philadelphia: Lipppincott Williams \& Wilkins; 2011.

3. Thompson JT. Advantages and limitations of small gauge vitrectomy. Surv Ophthalmol. 2011;56:162-172.

4. Territo C, Gieser JP, Wilson CA, Anand R. Influence of the cannulated vi trectomy system on the occurrence of iatrogenic sclerotomy retinal tears. Retina. 1997;17:430-433.

5. Covert DJ, Henry CR, Bhatia SK, et al. Intraoperative retinal tear formation and postoperative rhegmatogenous retinal detachment in transconjunctival cannulated vitrectomy systems compared with the standard 20-gauge system. Arch Ophthalmol. 2012;130(2):186-189.

6. Nishimura A, Kimura M, Okuda T, et al. Self-sealing cannula system for 20-gauge vitrectomy: outcome of 247 consecutive cases. Retina. 2008;28:778-781.

7. Early Treatment Diabetic Retinopathy Study Research Group. Fundus photographic risk factor for progression of diabetic retinopathy. ETDRS report number 10. Ophthalmology. 1991;98:786-806.

8. Eckardt C. Transconjunctival sutureless 23-gauge vitrectomy. Retina 2005;25:208-211.

9. Shimada H, Nakashizuka H, Mori R, et al. Expanded Indications for 25-gauge transconjunctival vitrectomy. Jpn J Ophthalmol. 2005;49: 397-401.

10. Sato T, Emi K, Bando H, et al. Retrospective Comparison of 25-gauge vitrectomy with 20-gauge vitrectomy in the repair of retinal detachment complicated with proliferative vitreoretinopathy. J Jpn Ophthalmol Soc. 2012;116:100-107.

11. Yamada K, Maeno T, Yamada M. Treatment of an oil-filled anterior chamber after microincision vitrectomy for recurrent retinal detachment. J Med Soc Toho. 2011;58(3):198-202.
Clinical Ophthalmology

\section{Publish your work in this journal}

Clinical Ophthalmology is an international, peer-reviewed journal covering all subspecialties within ophthalmology. Key topics include: Optometry; Visual science; Pharmacology and drug therapy in eye diseases; Basic Sciences; Primary and Secondary eye care; Patient Safety and Quality of Care Improvements. This journal is indexed on

\section{Dovepress}

PubMed Central and CAS, and is the official journal of The Society of Clinical Ophthalmology (SCO). The manuscript management system is completely online and includes a very quick and fair peer-review system, which is all easy to use. Visit http://www.dovepress.com/ testimonials.php to read real quotes from published authors. 